\title{
Altered Cultivar Resistance of Kimchi Cabbage Seedlings Mediated by Salicylic Acid, Jasmonic Acid and Ethylene
}

\author{
Young Hee Lee ${ }^{1}$, Sang Hee Kim² , Byung-Wook Yun ${ }^{3}$ and Jeum Kyu Hong ${ }^{1 *}$ \\ ${ }^{I}$ Department of Horticultural Science, Gyeongnam National University of Science and Technology (GNTech), 33 Dongjin- \\ ro, Jinju 660-758, Republic of Korea \\ ${ }^{2}$ Division of Plant Sciences, Christopher S. Bond Life Sciences Center and Interdisciplinary Plant Group, University of \\ Missouri, Columbia, Missouri, USA \\ ${ }^{3}$ Division of Plant Biosciences, School of Applied Biosciences, Kyungpook National University, Daegu 702-701, Republic \\ of Korea
}

(Received on June 10, 2014; Revised on July 7, 2014; Accepted on July 24, 2014)

Two cultivars Buram-3-ho (susceptible) and CR-Hagwang (moderate resistant) of kimchi cabbage seedlings showed differential defense responses to anthracnose (Colletotrichum higginsianum), black spot (Alternaria brassicicola) and black rot (Xanthomonas campestris pv. campestris, Xcc) diseases in our previous study. Defense-related hormones salicylic acid (SA), jasmonic acid (JA) and ethylene led to different transcriptional regulation of pathogenesis-related (PR) gene expression in both cultivars. In this study, exogenous application of SA suppressed basal defenses to $C$. higginsianum in the 1st leaves of the susceptible cultivar and cultivar resistance of the 2 nd leaves of the resistant cultivar. SA also enhanced susceptibility of the susceptible cultivar to $A$. brassicicola. By contrast, SA elevated disease resistance to $X c c$ in the resistant cultivar, but not in the susceptible cultivar. Methyl jasmonate (MJ) treatment did not affect the disease resistance to $C$. higginsianum and $X c c$ in either cultivar, but it compromised the disease resistance to $A$. brassicicola in the resistant cultivar. Treatment with 1-aminocyclopropane-1-carboxylic acid (ACC) ethylene precursor did not change resistance of the either cultivar to $C$. higginsianum and $X c c$. Effect of ACC pretreatment on the resistance to $A$. brassicicola was not distinguished between susceptible and resistant cultivars, because cultivar resistance of the resistant cultivar was lost by prolonged moist dark conditions. Taken together, exogenously applied SA, JA and ethylene altered defense signaling crosstalk to three diseases of anthracnose, black spot and black rot in a cultivar-

*Corresponding author.

Phone) +82-55-751-3251, FAX) +82-55-751-3257

E-mail) jkhong@gntech.ac.kr dependent manner.

Keywords : cultivar resistance, defense signaling, ethylene, kimchi cabbage, methyl jasmonate, salicylic acid

Cultivar resistance has been considered to be reliable and durable during crop production, and a variety of commercial cultivars and accessions were evaluated to determine genetic resources of disease resistance for economically important crops, such as pepper to anthracnose, rapeseed to Sclerotinia rot and watermelon to Phytophthora blight (Kim et al., 2010, 2013; Mei et al., 2012). More efficient screening methods including plant growth stage and inoculation procedure have been newly developed to find cultivar resistance of radish to clubroot (Jo et al., 2011). In vitro screening of anthracnose-resistant cassava cultivars on potato dextrose agar media amended with stem cortex extracts from different cassava cultivars corresponded well to cultivar resistance demonstrated in the greenhouse and under field conditions (Fokunang et al., 2002). A system of rapid seedling assay was also established for screening large numbers of onion cultivars using different isolates of Fusarium oxysporum f. sp. cepae that causes basal rot (Taylor et al., 2013). Resistant or moderately resistant cultivars can be employed in integrated disease management programs accompanied with other biological and chemical controls, because fungicides, plant defense activators, and antagonistic microbes were often more effective to reduce diseases in the resistant cultivars (Banani et al., 2014; Walters et al., 2011).

Cultivar resistances in many crops are closely associated with augmented cellular and biochemical defenses against pathogen infection compared to susceptible responses. 
Higher antioxidant enzyme activities of peroxidase, ascorbate peroxidase and glutathione reductase were reported to be found in anthracnose-resistant melon cultivar infected by Colletotrichum lagenarium (Ge et al., 2013). Anthracnose-resistant ripe blueberry fruits produces significantly more anthocyanins and non-anthocyanin flavonoids compared to susceptible ones (Miles et al., 2013). Recently transcriptome and proteome profiles of resistant cultivars from tomato and blueberry were investigated to reveal molecular and genetic clues of the resistance traits (Chen et al., 2013; Dahal et al., 2010; Miles et al., 2011).

In recent decades, crosstalk among distinct defense signaling pathways has been suggested in Arabidopsis plants inoculated by several bacterial and fungal pathogens triggering SA, JA and ethylene-mediated defense reactions. The hemibiotrophic bacterium Pseudomonas syringae pv. tomato and the biotrophic fungus Erysiphe orontii activated SA-dependent defense signaling pathways, whereas defenses to two necrotrophic fungi A. brassicicola and Botrytis cinerea were mainly mediated by JA/ethylene signaling in Arabidopsis (Lorenzo et al., 2003; Reuber et al., 1998; Thomma et al., 1998; Vlot et al., 2009). In general, SA and JA/ethylene-mediated defense signaling pathways are mutually antagonistic, and a reducing defense reaction(s) of one pathway often increases activation of the other (Boatwright and Pajerowska-Mukhtar, 2013; Derksen et al., 2013). Altered disease resistance of crops treated with SA, JA and ethylene as defense elicitors have been demonstrated. Accumulation of SA and JA increased with a distinct temporal profile during oilseed rape leaves by Sclerotinia sclerotiorum infection, and lesion development on the inoculated leaves was arrested by SA and JA treatments (Wang et al., 2012). However, defense signaling crosstalk in crops during pathogenesis or cell deathmediated resistance has been rarely described.

In our previous study, two cultivars of kimchi cabbage (Brassica rapa var. glabra Regel) seedlings demonstrated differential disease resistance to infections by the hemibiotrophic fungus $C$. higginsianum, the necrotrophic $A$. brassicicola and the hemibiotrophic bacterium Xcc (Lee and Hong, 2014). PR gene expressions were much more induced in the resistant cultivar CR-Hagwang treated with SA compared to the susceptible cultivar Buram-3-ho. In this study, we aimed to investigate whether exogenous application of the three defense-related chemicals SA, MJ and ACC altered the disease resistance of susceptible and resistant cultivars to anthracnose, black spot and black rot diseases or not. This may provide better understanding of defense signaling crosstalk in the two different kimchi cabbage cultivars, and further suggest agriculturally practical approaches to improve broad spectrum disease resistance. Pathogen growth, plant inoculation and disease assessment were performed as previously described (Lee and Hong, 2014). To analyze pretreatment effect of the defense hormones on the disease resistance of kimchi cabbage seedlings, SA $(0.5 \mathrm{mM}), \mathrm{MJ}(0.1 \mathrm{mM})$ and ACC $(0.1$ $\mathrm{mM}$ ) solutions were sprayed on foliage $3 \mathrm{~h}, 3 \mathrm{~h}$ and $9 \mathrm{~h}$, respectively, prior to fungal and bacterial inoculations, at which most PR gene expressions in the resistant cultivar were highly activated in our previous study (Lee and Hong, 2014). All experiments were repeated four times. Pretreatment with SA, MJ and ACC differentially modulated disease resistance to the fungal and bacterial infections in susceptible and resistant cultivars of kimchi cabbage seedlings.

Changes in anthracnose resistance derived by SA pretreatment were differently demonstrated in the 1st and 2nd leaves of both cultivars (Fig. 1A). Basal disease resistance to $C$. higginsianum was reduced by SA in the 1 st leaves of susceptible cv. Buram-3-ho, but not in the resistant cv. CRHagwang. In the 2nd leaves, SA pretreatment enhanced susceptibility only in the resistant cultivar CR-Hagwang, but no change occurred in disease resistance of susceptible cv. Buram-3-ho. Synergistic and/or antagonistic interactions between several defense signaling pathways have been shown to confer disease resistance to invading pathogens (Gimenez-Ibanez and Solano, 2013; Pieterse et al., 2009). SA may antagonistically suppress the JA-mediated defenses in kimchi cabbage seedlings as demonstrated in several plant species including Arabidopsis and tomato plants. SA application enlarged the decayed area of tomato fruit infected by $C$. coccodes, whereas JA reduced the lesion area, indicating that SA lowered JA-mediated defenses of tomato fruits to C. coccodes (Alkan et al., 2012).

SA-enhanced disease susceptibility was also observed in the susceptible kimchi cabbage cultivar inoculated with $A$. brassicicola. Pretreatment of susceptible cv. Buram-3-ho with SA heightened susceptibility to $A$. brassicicola infection, but did not change the disease resistance of resistant cv. CR-Hagwang (Fig. 1B). SA treatment reduced JAinducible $P D F 1.2$ gene expression and increased lesion size in Arabidopsis inoculated by A. brassicicola suggesting antagonistic interaction between SA and JA during the resistance response (Leon-Reyes et al., 2009, 2010; Spoel et al., 2007). Disease lesions were progressive much more in SA-pretreated tomato leaves inoculated by Alternaria solani (El Rahman et al., 2012). These results may be due to suppression of JA-dependent defenses by SA treatment in susceptible host plants. However, SA was defenseinducing elicitor in pear fruit inoculated with Alternaria 

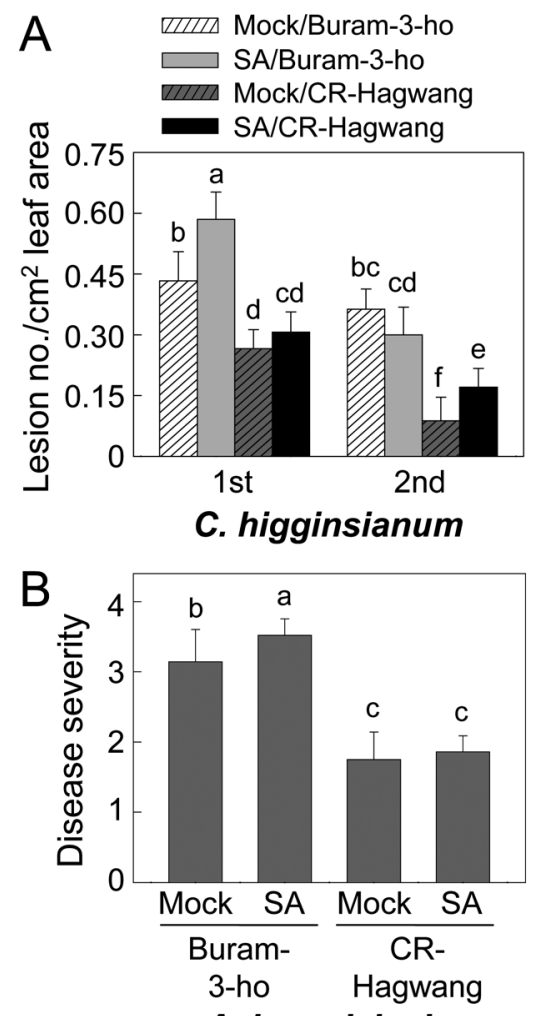

A. brassicicola

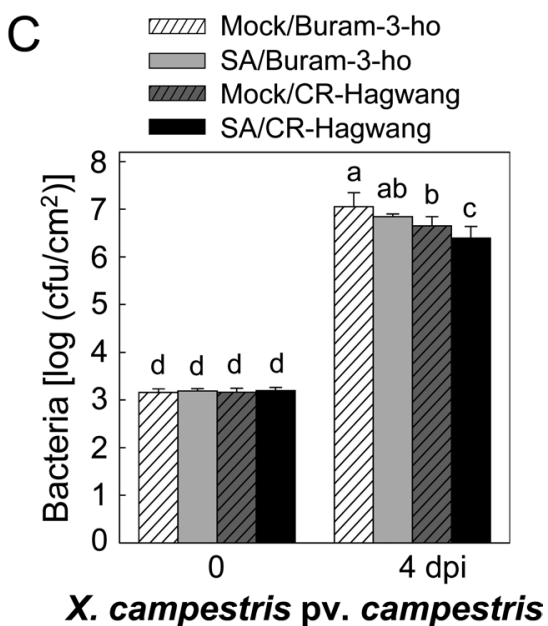

Fig. 1. Altered disease resistance of kimchi cabbage seedlings to fungal and bacterial infections in susceptible (cv. Buram-3ho) and resistant (cv. CR-Hagwang) plants pretreated with 0.5 $\mathrm{mM}$ of salicylic acid (SA). (A) Lesion numbers on the inoculated leaves of both kimchi cabbage cultivars pretreated with SA were counted after $C$. higginsianum inoculation. 1st, 1st true leaves; 2nd, 2nd true leaves. (B) Black spot disease severity of both kimchi cabbage cultivars pretreated with SA. (C) Xcc bacterial growth in both kimchi cabbage cultivars pretreated with SA. dpi, days post-inoculation. Data points are the mean \pm standard errors of four independent experiments. The means with different letter were significant at the $5 \%$ level as determined by Duncan's multiple range test. alternata (Tian et al., 2006). We suggested that the resistant cv. CR-Hagwang might activate SA, JA and ethylenemediated defenses all together to combat pathogens having different infection strategies and to achieve broad spectrum disease resistance in our previous study (Lee and Hong, 2014). Although SA application more or less reduced disease resistance to $C$. higginsianum and $A$. brassicicola in the susceptible cultivar, it did not occur in the resistant cultivar. Exogenously applied SA maybe cannot suppress JAand ethylene-mediated defenses in the resistant cultivar, because JA and ethylene-signaling were also highly upregualted by these fungal infections.

In this study, we also evaluated disease response of $2 \mathrm{nd}$ leaves of the seedlings pretreated with SA. Enhanced disease susceptibility in the susceptible cultivar was not found in the 2 nd leaves infected by $C$. higginsianum, whereas high level of resistance in the 2 nd leaves was decreased by SA. These are contrary to the facts found in the 1st leaves. SA-triggered differential molecular responses in the 1st and 2nd leaves against $C$. higginsianum infection remain to be elucidated.

In contrast, SA application led to enhanced disease resistance to $X c c$ only in the resistant cv. CR-Hagwang, but not in the susceptible cv. Buram-3-ho (Fig. 1C). This suggests that SA-enhanced disease resistance was only marginally activated in the resistant cultivar. Resistant cv. CRHagwang may have sensitive mechanisms to endogenously activated SA signals induced by Xcc infection as well as may produce more SA by Xcc infection. It is in line with the fact that SA application activated an array of PR genes related to resistance to Xcc preferentially in cv. CR-Hagwang (Lee and Hong, 2014). SA-mediated induced resistance has been often demonstrated in various plant species infected by viral (Cucumber mosaic virus, Turnip crinkle virus), bacterial (Erwinia carotovora subsp. carotovora) and fungal (Erysiphe cichoracearum, Fusarium oxysporum f. sp. lycopersici, Gaeumannomyces graminis var. tritici) pathogens (Mandal et al., 2009; Mayers et al., 2005; Sari and Etebarian., 2009; Shang et al., 2011; Vimala and Suriachandraselvan, 2009). Current study is the first report of SA-induced disease resistance of kimchi cabbage plants to a bacterial infection. Meanwhile, it is noteworthy that SA could not induce resistance to $X c c$ in the susceptible cultivar of kimchi cabbage seedlings. Pivotal components for SA-mediated defense activation might be deficient in the susceptible cultivar of kimchi cabbage seedlings as demonstrated in a variety of SA-related Arabidopsis mutants (Moreau et al., 2012; Ng et al., 2011).

MJ treatment reduced lesion size on peach fruits inoculated by postharvest diseases Monilinia fructicola and Pen- 

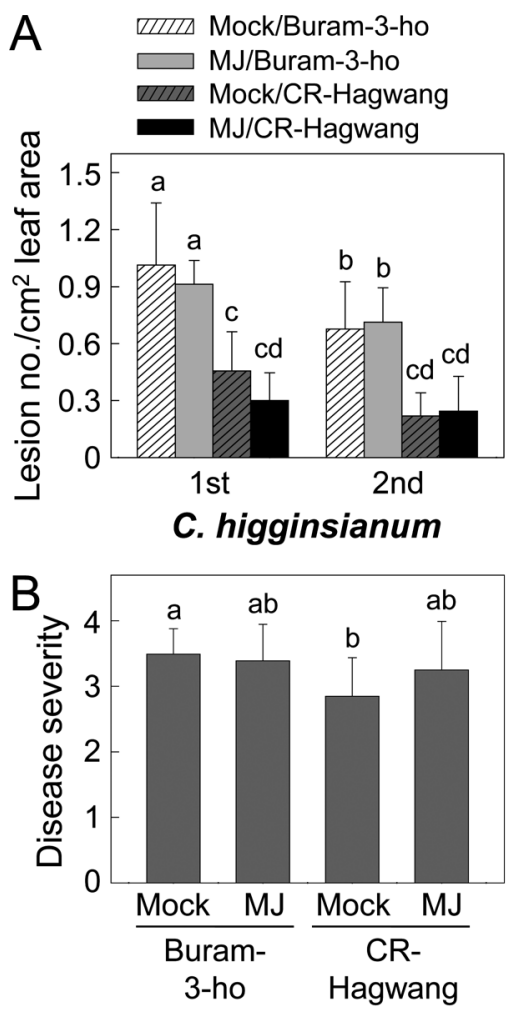

A. brassicicola

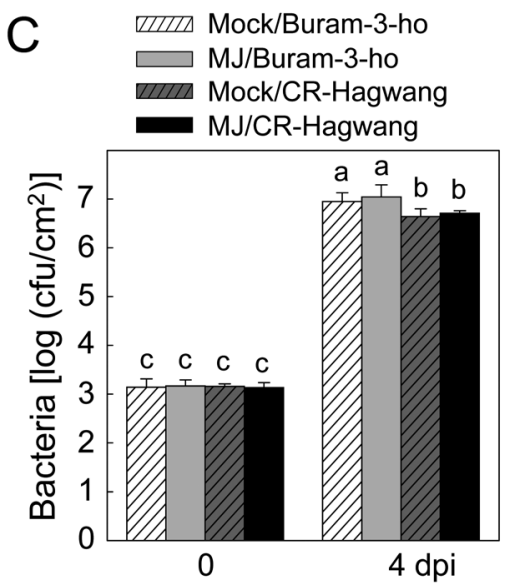

$X$. campestris pv. campestris

Fig. 2. Altered disease resistance of kimchi cabbage seedlings to fungal and bacterial infections in susceptible (cv. Buram-3-ho) and resistant (cv. CR-Hagwang) plants pretreated with $0.1 \mathrm{mM}$ of methyl jasmonate (MJ). (A) Lesion numbers on the inoculated leaves of both kimchi cabbage cultivars pretreated with methyl jasmonate (MJ) were counted after $C$. higginsianum inoculation. 1st, 1st true leaves; 2nd, 2nd true leaves. (B) Black spot disease severity of both kimchi cabbage cultivars pretreated with MJ. (C) $X c c$ bacterial growth in both kimchi cabbage cultivars pretreated with MJ. dpi, days post-inoculation. Data points are the mean \pm standard errors of four independent experiments. The means with different letter were significant at the $5 \%$ level as determined by Duncan's multiple range test. icillium expansum, but permitted cell-to-cell movement of Tobacco mosaic virus in tobacco leaves (Oka et al., 2013; Yao and Tian, 2005). Ethylene conferred disease resistance of tobacco plants to $C$. destructivum, but disease susceptibility to loquat fruit to C. acutatum (Cao and Zheng, 2010; Chen et al., 2003). These findings have suggested that involvement of JA and ethylene in plant defenses and protections occurred differently in diverse conditions. Pretreatment with MJ and ACC did not affect the disease resistance to $C$. higginsianum, A. brassicicola and the bacterial $X c c$ infection in either kimchi cabbage cultivar (Figs. 2 and 3). Surprisingly, cultivar resistance of CR-Hagwang to $A$. brassicicola was diminished even in the mock-inoculated seedlings (Figs. 2B and 3B). In the present study, we placed both kimchi cabbage seedling cultivars under moist dark conditions using black plastic covers for $3 \mathrm{~h}$ and $9 \mathrm{~h}$ to investigate effect of MJ and ACC, respectively, and additional moist dark conditions were treated for the fungal disease development after inoculation. Interestingly, longer exposure to the moist dark conditions prior to A. brassicicola infection seemed to further compromise innate cultivar resistance of cv. CR-Hagwang. Difference in disease severity of the two cultivars to A. brassicicola was reduced by 3 h-mock treatment and 9 h-moist/dark periods completely eliminated black spot resistance of cv. CR-Hagwang. It is maybe caused by enhanced vulnerability of the host plants to challenging pathogens via microclimate change like limited light and increased humidity on the plant surface. Adverse effect of environmental changes on the establishment of disease resistance of cv. CR-Hagwang should be further elucidated. Cultivar CR-Hagwang contained relatively higher chlorophyll contents accumulated (Lee and Hong, 2014). We suppose that it could confer at least in part disease resistance of $\mathrm{cv}$. CR-Hagwang shown in our previous study, although more sophisticated experimental evidence needed (Lee and Hong, 2014). Reduced chlorophyll contents in cv. CR-Hagwang by inhibition of photosynthesis could lead to vulnerability status of kimchi cabbage seedlings to $A$. brassicicola infection. Closed lids in this study may affect ethylene concentration in the ambient condition of the kimchi seedlings and negatively regulate disease resistance to $A$. brassicicola in cv. CR-Hagwang (Leon-Reyes et al., 2009). By contrast, prolonged moist dark conditions did not severely affect cultivar resistance of cv. CR-Hagwang against $C$. higginsianum, suggesting differentiated defense signaling pathways against both fungal pathogens.

Disease resistance to Xcc was not altered by JA and ethylene treatment in both susceptible and resistant cultivars of kimchi cabbage seedlings. This result correlates with the facts that SA diminished JA-induced PDF1.2 gene expres- 


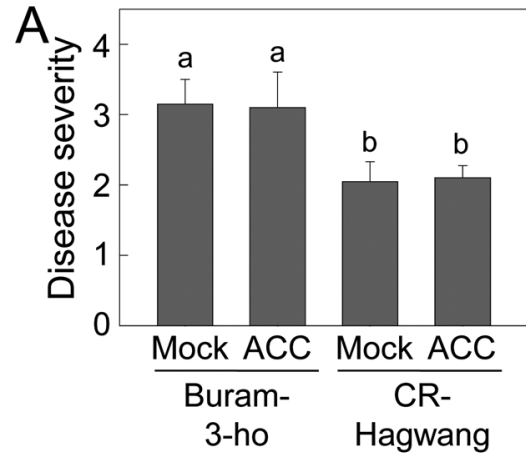

C. higginsianum

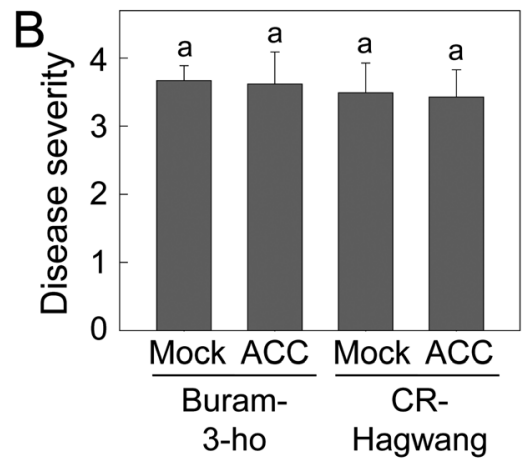

A. brassicicola

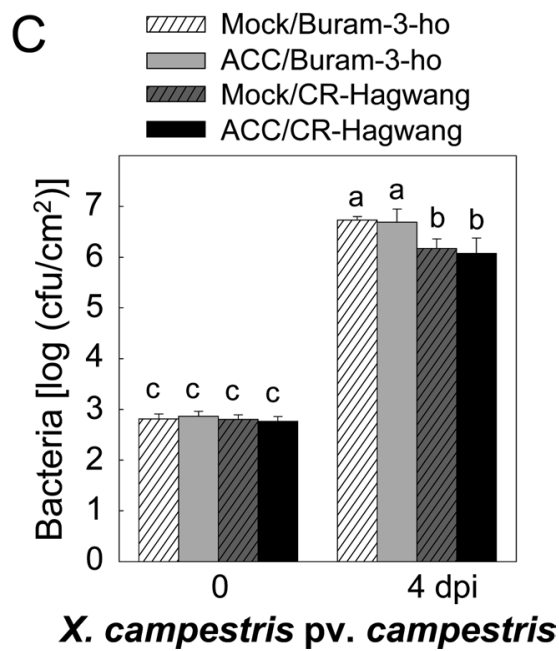

Fig. 3. Altered disease resistance of kimchi cabbage seedlings to fungal and bacterial infections in susceptible (cv. Buram-3-ho) and resistant (cv. CR-Hagwang) plants pretreated with $0.1 \mathrm{mM}$ of 1-aminocyclopropane-1-carboxylic acid (ACC). (A) Anthracnose disease severity of the inoculated seedlings pretreated with ACC was evaluated. (B) Black spot disease severity of both kimchi cabbage cultivars pretreated with ACC. (C) Xcc bacterial growth in both kimchi cabbage cultivars pretreated with ACC. dpi, days post-inoculation. Data points are the mean \pm standard errors of four independent experiments. The means with different letter were significant at the 5\% level as determined by Duncan's multiple range test. sion but JA could not suppress SA-inducible $P R 1$ gene expression (Leon-Reyes et al., 2009; Spoel et al., 2007).

In recent years, abscisic acid (ABA) and nitric oxide (NO) as novel plant defense regulators have been integrated into well-known defense signaling crosstalk associated with SA, JA and ethylene (Audenaert et al., 2002; de Torres Zabala et al., 2009; Leitner et al., 2009; Mur et al., 2013). Involvement of these small molecules in the disease resistance of kimchi cabbage seedlings remains to be elucidated.

In conclusion, defense hormones SA, JA and ethylene may alter innate immunity of susceptible and resistant cultivars of kimchi cabbage against pathogen infections with different invasion strategies. The current study will provide the molecular and genetic bases to gain more insight on defense signaling in disease susceptibility and resistance of kimchi cabbage.

\section{Acknowledgments}

We are grateful to Ms. Sharon Pike (University of Missouri, USA) for the helpful discussion and critical reviewing our manuscript. This work was carried out with the support of "Cooperative Research Program for Agriculture Science \& Technology Development (Project No. PJ008211)", Rural Development Administration, Republic of Korea.

\section{References}

Alkan, N., Fluhr, R. and Prusky, D. 2012. Ammonium secretion during Colletotrichum coccodes infection modulates salicylic acid and jasmonic acid pathways of ripe and unripe tomato fruit. Mol. Plant-Microbe Interact. 25:85-96.

Audenaert, K., De Meyer, G. B. and Höfte, M. M. 2002. Abscisic acid determines basal susceptibility of tomato to Botrytis cinerea and suppresses salicylic acid-dependent signaling mechanisms. Plant Phyiol. 128:491-501.

Banani, H., Roatti, B., Ezzahi, B., Giovannini, O., Gessler, G., Pertot, I. and Perazzolli, M. 2014. Characterization of resistance mechanisms activated by Trichoderma harzianum T39 and benzothiadiazole to downy mildew in different grape cultivars. Plant Pathol. 63:334-343.

Boatwright, J. L. and Pajerowska-Mukhtar, K. 2013. Salicylic acid: an old hormone up to new tricks. Mol. Plant Pathol. 14:623-634.

Cao, S. and Zheng, Y. 2010. Effect of 1-methylcyclopropane on anthracnose rot caused by Colletotrichum acutatum and disease resistance in loquat fruit. J. Sci. Food Agric. 90:22892294.

Chen, N., Goodwin, P. H. and Hsiang, T. 2003. The role of ethylene during the infection of Nicotiana tabacum by Colletotrichum destructivum. J. Exp. Bot. 54:2449-2456. 
Chen, T., Lv, Y., Zhao, T., Li, N., Yang, Y., Yu, W., He, X., Liu, T. and Zhang, B. 2013. Comparative transcriptome profiling of a resistant vs. susceptible tomato (Solanum lycopersicum) cultivar in response to infection by tomato yellow leaf curl virus. PLOS ONE 8:e80816.

Dahal, D., Pich, A., Braun, H. P. and Wydra, K. 2010. Analysis of cell wall proteins regulated in stem of susceptible and resistant tomato species after inoculation with Ralstonia solanacearum: a proteomic approach. Plant Mol. Biol. 73:643658.

Derksen, H., Rampitsch, C. and Daayf, F. 2013. Signaling crosstalk in plant disease resistance. Plant Sci. 207:79-87.

de Torres Zabala, M., Bennett, M. H., Truman, W. H. and Grant, M. R. 2009. Antagonism between salicylic acid and abscisic acid reflects early host-pathogen conflict and moulds plant defence responses. Plant J. 59:375-386.

El Rahman, T. A., El Oirdi, M., Gonzalez-Lamothe, R. and Bouarab, K. 2012. Necrotrophic pathogens use the salicylic acid signaling pathway to promote disease development in tomato. Mol. Plant-Microbe Interact. 25:1584-1593.

Fokunang, C. N., Dixon, A. G. O., Ikotun, T., Akem, C. N. and Tembe, E. A. 2002. Rapid screening method of cassava cultivars for resistance to Colletotrichum gloeosporioides f. sp. manihotis. J. Phytopathol. 150:6-12.

Ge, Y., Bi, Y. and Guest, D. I. 2013. Defence responses in leaves of resistant and susceptible melon (Cucumis melo L.) cultivars infected with Colletotrichum lagenarium. Physiol. Mol. Plant Pathol. 81:13-21.

Gimenez-Ibanez, S. and Solano, R. 2013. Nuclear jasmonate and salicylate signaling and crosstalk in defense against pathogens. Front. Plant Sci. 4:1-11.

Jo, S.-J., Jang, K. S., Choi, Y. H., Kim, J.-C. and Choi, G. J. 2011. Development of convenient screening method for resistant radish to Plasmodiophora brassicae. Res. Plant Dis. 17:161168. (in Korean)

Kim, J.-S., Jee, H.-J., Gwag, J.-G., Kim, C.-K. and Shim, C.-K. 2010. Evaluation on red pepper germplasm lines (Capsicum spp.) for resistance to anthracnose caused by Colletotrichum acutatum. Plant Pathol. J. 26:273-279.

Kim, M.-J., Shim, C.-K., Kim, Y.-K., Jee, H.-J., Hong, S.-J., Park, J.-H. and Han, E.-J. 2013. Evaluation of watermelon germplasm for resistance to Phytophthora blight caused by Phytophthora capsici. Plant Pathol. J. 29:87-92.

Lee, Y. H. and Hong, J. K. 2014. Differential defence responses of susceptible and resistant kimchi cabbage cultivars to anthracnose, black spot and black rot diseases. Plant Pathol. DOI: $10.1111 / \mathrm{ppa} / 12262$.

Leitner, M., Vandelle, E., Gaupels, F., Bellin, D. and Delledonne, M. 2009. NO signals in the haze: Nitric oxide signalling in plant defence. Curr. Opin. Plant Biol. 12:451-458.

Leon-Reyes, A., Du, Y., Koornneef, A., Proietti, S., Körbes, A. P., Memelink, J., Pieterse, C. M. J. and Ritsema, T. 2010. Ethylene signaling renders the jasmonate response of Arabidopsis insensitive to future suppression by salicylic acid. Mol. Plant-
Microbe Interact. 23:187-197.

Leon-Reyes, A., Spoel, S. H., De Lange, E. S., Abe, H., Kobayashi, M., Tsuda, S., Millenaar, F. F., Welschen, R. A. M., Ritsema, T. and Pieterse, C. M. J. 2009. Ethylene modulates the role of NONEXPRESSOR OF PATHOGENESIS-RELATED GENES1 in cross talk between salicylate and jasmonate signaling. Plant Physiol. 149:1797-1809.

Lorenzo, O., Piqueras, R., Sanchez-Serrano, J. J. and Solano, R. 2003. ETHYLENE RESPONSE FACTOR1 integrates signals from ethylene and jasmonate pathways in plant defense. Plant Cell 15:165-178.

Mandal, S., Mallick, N. and Mitra, A. 2009. Salicylic acidinduced resistance to Fusarium oxysporum f. sp. lycopersici. Plant Physiol. Biochem. 47:642-649.

Mayers, C. N., Lee, K.-C., Moore, C. A., Wong, S.-M. and Carr, J. P. 2005. Salicylic acid-induced resistance to Cucumber mosaic virus in squash and Arabidopsis thaliana: contrasting mechanisms of induction and antiviral action. Mol. PlantMicrobe Interact. 18:428-434.

Mei, J., Wei, D., Disi, J. O., Ding, Y., Liu, Y. and Qian, W. 2012. Screening resistance against Sclerotinia sclerotiorum in Brassica crops with use of detached stem assay under controlled environment. Eur. J. Plant Pathol. 134:599-604.

Miles, T. D., Day, B. and Schilder, A. C. 2011. Identification of differentially expressed genes in a resistant versus susceptible blueberry cultivar after infection by Colletotrichum acutatum. Mol. Plant Pathol. 12:463-477.

Miles, T. D., Vandervoort, C., Nair, M. G. and Schilder, A. C. 2013. Characterization and biological activity of flavonoids from ripe fruit of an anthracnose-resistant blueberry cultivar. Physiol. Mol. Plant Pathol. 82:8-16.

Moreau, M., Tian, M. and Klessig, D. F. 2012. Salicylic acid binds NPR3 and NPR4 to regulate NPR1-dependent defense responses. Cell Res. 22:1631-1633.

Mur, L. A. J., Prats, E., Pierre, D., Hall, M. A. and Hebelstrup, K. H. 2013. Integrating nitric oxide into salicylic acid and jasmonic acid/ethylene plant defense pathways. Front. Plant Sci. $4: 125$.

Ng, G., Seabolt, S., Zhang, C., Salimian, S., Watkins, T. A. and Lu, H. 2011. Genetic dissection of salicylic acid-mediated defense signaling networks in Arabidopsis. Genetics 189:851859.

Oka, K., Kobayashi, M., Mitsuhara, I. and Seo, S. 2013. Jasmonic acid negatively regulates resistance to Tobacco mosaic virus in tobacco. Plant Cell Physiol. 54:1999-2010.

Pieterse, C. M. J., Leon-Reyes, A., van der Ent, S. and van Wees, S. C. M. 2009. Networking by small-molecule hormones in plant immunity. Nat. Chem. Biol. 5:308-317.

Reuber, T. L., Plotnikova, J. M., Dewdney, J., Rogers, E. E., Wood, W. and Ausubel, F. M. 1998. Correlation of defense gene induction defects with powdery mildew susceptibility in Arabidopsis enhanced disease susceptibility mutants. Plant J. 16:473-485.

Sari, E. and Etebarian, H. R. 2009. Concentration-dependent ef- 
fect of salicylic acid application on wheat seedling resistance to take-all fungus, Gaeumannomyces graminis var. tritici. Phytoparasitica 37:67-76.

Shang, J., Xi, D.-H., Xu, F., Wang, S.-D., Cao, S., Xu, M.-Y., Zhao, P.-P., Wang, J.-H., Jia, S.-D., Zhang, Z.-W., Yuan, S. and Lin, H.-H. 2011. A broad-spectrum, efficient and nontransgenic approach to control plant viruses by application of salicylic acid and jasmonic acid. Planta 233:299-308.

Spoel, S. H., Johnson, J. S. and Dong, X. 2007. Regulation of tradeoffs between plant defenses and against pathogens with different lifestyles. Proc. Natl. Acad. Sci. USA 104:1884218847.

Taylor, A., Vagany, V., Barbara, D. J., Thomas, B., Pink, D. A. C., Jones, J. E. and Clarkson, J. P. 2013. Identification of differential resistance to six Fusarium oxysporum f. sp. cepae isolates in commercial onion cultivars through the development of a rapid seedling assay. Plant Pathol. 62:103-111.

Thomma, B. P., Eggermont, K., Penninckx, I. A., Mauch-Mani, B., Vogelsang, R., Cammue, B. P. and Broekaert, W. F. 1998. Separate jasmonate-dependent and salicylate-dependent defense-response pathways in Arabidopsis are essential for resistance to distinct microbial pathogens. Proc. Natl. Acad.
Sci. USA 95:15107-15111.

Tian, S., Wan, Y., Qin, G. and Xu, Y. 2006. Induction of defense responses against Alternaria rot by different elicitors in harvested pear fruit. Appl. Microbiol. Biotechnol. 70:729-734.

Vimala, R. and Suriachandraselva, M. 2009. Induced resistance in bhendi against powdery mildew by foliar application of salicylic acid. J. Biopest. 2:111-114.

Vlot, A. C., Dempsey, D. A. and Klessig, D. F. 2009. Salicylic acid, a multifaceted hormone to combat disease. Annu. Rev. Phytopathol. 47:177-206.

Walters, D. R., Havis, N. D., Paterson, L., Taylor, J. and Walsh, D. J. 2011. Cultivar effects on the expression of induced resistance in spring barley. Plant Dis. 95:595-600.

Wang, Z., Tan, X., Zhang, Z., Gu, S., Li, G. and Shi, H. 2012. Defense to Sclerotinia sclerotiorum in oilseed rape is associated with the sequential activations of salicylic acid signaling and jasmonic acid signaling. Plant Sci. 184:75-82.

Yao, H. J. and Tian, S. P. 2005. Effects of a biocontrol agent and methyl jasmonate on postharvest diseases of peach fruit and the possible mechanisms involved. J. Appl. Microbiol. 98:941-950. 